Results In the last 10 years, 11 babies had a documented diagnosis of Ebstein's anomaly. Ten of these were antenatally diagnosed. Four were born prematurely ( $<37$ weeks). Eight babies required resuscitation at birth and nine babies required intubation within the first 24 hours of life. Many of the babies had other significant co-morbidities including: hydrops fetalis, seizure activity, ventriculomegaly, trisomy 21 and persistent pulmonary hypertension (PPHN). Two babies also underwent therapeutic hypothermia. All of the babies born prematurely died within the neonatal period. Of the seven term babies with Ebstein's anomaly, three were discharged home, two were transferred to the Regional Paediatric Cardiology unit and two died on the neonatal unit. The two babies that were transferred the Regional Paediatric Cardiology unit subsequently died. The three surviving infants continue to be followed up. One of these patients is now seven years old and has not required any surgical intervention.

Conclusion In our experience, Ebstein's anomaly was uniformly fatal in babies born prematurely. Furthermore, in term babies the survival was $43 \%$. The overall survival of Ebstein's anomaly presenting in the neonatal period in our cohort was $27 \%$. Although this is an outcome of a small cohort of neonates with a rare congenital heart disease, it is important that parents are counselled appropriately antenatally so that they are adequately prepared for the potentially poor outlook of babies born with Ebstein's anomaly in the neonatal period.

\section{G22(P) CHILD WITH HYPERTROPHIC CARDIOMYOPATHY DUE TO PRIMARY CARNITINE DEFICIENCY (PCD)}

Y Kodeeswaran, S Rajendran. Paediatrics, Luton and Dunstable Hospital, Luton, UK

\subsection{6/archdischild-2020-rcpch. 11}

Introduction Primary carnitine deficiency (PCD) is a rare autosomal recessive disorder of fatty acid oxidation, in which the transport of carnitine into cells is impaired. Carnitine deficiency blocks the oxidation of long-chain fatty acids in the mitochondria that leads to heart and hepatic disease, myopathy, nonketotic hypoglycaemia, and neurological complications. Case Report 7 month old child presented with 3 day history of vomiting.

$\mathrm{He}$ was born at term to non-consanguineous parents. He had returned to the UK recently, after a 4 month stay in India. Whilst in India he had had 2 hospital admissions with vomiting: one of which required admission to PICU with hypoglycaemia and metabolic acidosis, diagnosed as gastroenteritis and the next was for UTI.

$\mathrm{O} / \mathrm{E}$ : Baby was drowsy, with evidence of subcostal recession and hepatomegaly.

Started on antibiotics for possible sepsis, given saline bolus, and started on maintenance fluids. He later developed hypoglycaemia, metabolic acidosis, and fluctuating conciousness. After a discussion with the metabolic team, he was intubated and transferred to PICU.

Investigations Ammonia levels increased from 164 to 340 micromol/L in 6 hours, high ALT and deranged clotting. Free carnitine $<1$ umol/l.

Initial ECHO showed mild left ventricular hypertrophy. $\mathrm{He}$ had cardiopulmonary arrest on day 3 of PICU admission and consequently needed ECMO for 4 days. He was noted to have hypertrophic cardiomyopathy with left ventricular dysfunction. He required multiple ionotropes and nitric oxide and also developed intracranial bleeding and subsequent seizures, requiring Levetiracetam. Other complications included AKI ( required CVVH), liver failure, MRSA Bacteremia and E-Coli septicemia, which were treated.

Significant improvement was noted following levocarnitine replacement- he made a good recovery and was discharged home.

Discussion Primary carnitine deficiency (PCD) usually presents with cardiomyopathy and muscle weakness and is one of the treatable causes of cardiomyopathy.

Treatment of this disorder with L-carnitine is highly effective in correcting cardiomyopathy and muscle weakness.

Child may be asymptomatic until they develop signs of cardiomyopathy and congestive heart failure. Cardiac function does not respond to inotropes or diuretics. If no carnitine is supplemented, progressive heart failure eventually leads to death.

\section{G23(P) A 10 YEAR EXPERIENCE OF NEONATES WITH TRUNCUS ARTERIOSUS}

M Jagga, EL Stockley, T Desai, SV Rasiah. Birmingham Women's and Children's NHS Foundation Trust, Birmingham, UK

10.1136/archdischild-2020-rcpch.12

Background Congenital heart defect affects around 10 in a 1000 live born babies. Truncus arteriosus is a rare conotruncal defect in which a common arterial trunk supplies systemic, pulmonary and coronary circulation. The incidence of truncus arteriosus is quoted between $1-2 \%$ of all congenital heart malformations.

Aim To evaluate the initial neonatal management and outcomes of newborns with truncus arteriosus.

Method We conducted a retrospective review of all newborns with truncus arteriosus admitted to our neonatal unit between 01/04/2009 and 31/03/2019. Data were collected from Badger and Cardiology databases to evaluate the initial neonatal management and long-term outcomes of this cohort of patients.

Results Sixteen newborns with truncus arteriosus were admitted to our neonatal unit in the last 10 years. Their mean gestational age was 36 weeks with a mean birth weight of 2407 grams. Cardiac defects were detected prenatally in 13 patients (82\%). Three babies were diagnosed postnatally. One following failed pulse oximetry screening requiring non-invasive respiratory support and 2 diagnosed following on-going respiratory support on echocardiography. Respiratory support was initiated within the first 24 hours of life in 8 patients; these included all patients with a postnatal diagnosis of truncus arteriosus. Four patients required mechanical ventilation. Whilst in the neonatal intensive care unit, 3 babies developed heart failure and were treated with diuretics and 2 developed necrotising enterocolitis which was managed conservatively. Median length of stay before transfer to our local surgical cardiology centre was 3 days. Fourteen patients (87\%) underwent common arterial trunk repair. $11(78 \%)$ babies survived to date. Two patients died in the initial post-operative period and one patient at 14 months of age. The remaining two patients did not receive any surgical intervention and subsequently died (4 and 11 days old).

Conclusion Truncus arterisus is a rare conotruncal defect with the majority being detected antenatally in our cohort. They 